\title{
Prophylaxis of Rheumatic Fever/Rheumatic Heart Disease-Penicillin or Azithromycin, Who Wins?
}

\author{
Arati Lalchandani ${ }^{1}$ Taruni Lalchandani ${ }^{2} \quad$ Lubna Ahmad $^{3}$ Devarth Lalchandani ${ }^{4}$ \\ 1Professor of Medicine, GSVM Medical College, Kanpur, India \\ 2ENT Department, Vivekananda Polyclinic, Lucknow, India \\ ${ }^{3}$ Ophthalmology Department, GSVM Medical College, Kanpur, India

\begin{abstract}
Address for correspondence Devarth Lalchandani, MS, Professor of Medicine, GSVM Medical College, Kanpur 7/154 Swarup Nagar,
\end{abstract} \\ Kanpur, 208002, India (e-mail: devarthlalchandani@gmail.com).
}

${ }^{4}$ ENT Department, GSVM Medical College, Kanpur, India

Ind J Car Dis Wom 2021;6:42-45.

\begin{abstract}
Keywords

- Azithromycin

- penicillin

- ARMOR

For eradication of rheumatic fever (RF)/rheumatic heart disease (RHD), we must have a simplified diagnosis, and a treatment which is painless, easily available and safe; prophylaxis must be painless, safe, easily available, readily administered, and comprising a weekly oral single dose.

Arati's regime for management of RF/RHD (ARMOR) consists of diagnosis and management of RF/RHD in today's context in a very easy and simplified way.

ARMOR criteria: Arthritis or arthralgia with typical features suggestive of RF, carditis or cardiac involvement, typical of RF or RHD, and echocardiographic evidence of rheumatic heart valve involvement should essentially be the criteria to diagnose RF and RHD with high specificity and sensitivity.

With regard to treatment of RF/RHD, we need a drug which is highly efficacious against Group A Beta Hemolytic Streptococcus (GABHS), which is the causative agent for primary prevention and treatment and secondary prophylaxis.

The best drug discovered, to date, for GABHS is azithromycin.

ARMOR for primary prevention, treatment and secondary prophylaxis of RF/RHD is as follows:

Azithromycin must be given in a dose of $500 \mathrm{mg} 1$ tablet daily for 5 days, followed by 1 tablet once a week for 1 year.

Penicillin for treatment and prophylaxis must be given up due to its lack of availability, side effects, risk of anaphylaxis, parenteral preparation, hazards of administration, need for sensitivity test each time, etc. and replaced by azithromycin.
\end{abstract}

The more than seven decades old Jones criteria for diagnosis and management of rheumatic fever (RF) should only form a skeleton base for today's way of diagnosis and management of RHD and not copied ad verbatim.

ARMOR for treatment and prophylaxis: Acute rheumatic fever (ARF) begins with respiratory tract infection (RTI) or sore throat caused by group A $\beta$-hemolytic streptococci (GABHS). Treatment and prophylaxis should be started on suspicion of RF/rheumatic heart disease (RHD); therefore, diagnostic criteria must include echo Doppler study. For decades, the drug in use, such as penicillin, have failed to check the disease, prevent the disease, reduce the severity, or significantly alter the course of the disease.

A study by the International RF study group from 11 countries in 1790 patients showed recurrence in 45 percent of patients who received benzathine penicillin prophylaxis compared with $11.5 \%$ who failed to comply. The inference was that recurrence might occur even with published online

April 13, 2021
DOI https://doi.org/

$10.1055 / \mathrm{s}-0041-1728220$
(C)2021. Women in Cardiology and Related Sciences.

This is an open access article published by Thieme under the terms of the Creative Commons Attribution-NonDerivative-NonCommercial-License, permitting copying and reproduction so long as the original work is given appropriate credit. Contents may not be used for commercial purposes, or adapted, remixed, transformed or built upon. (https://creativecommons.org/licenses/by-nc-nd/4.0/).

Thieme Medical and Scientific Publishers Pvt. Ltd. A-12, 2nd Floor, Sector 2, Noida-201301 UP, India 
best prophylaxis, and compliance is essential but inadequate with benzathine penicillin $\mathrm{G}$ (BPG). ${ }^{1}$

For more than five decades, the primary treatment and secondary prophylaxis of RF/RHD has been BPG injection, 1.2 million units after a sensitivity test and repeated every 21 days in the same dose with inconsistent results.

Despite the discovery of a drug like azithromycin, which has a miraculous curative effect on the causative agent of $\mathrm{RF} / \mathrm{RHD}$, that is, group A streptococcus, it has not found a place in textbooks in the chapter on RF/RHD. azithromycin is a class of antibiotics called Azalides. The chemical name of azithromycin is 9-deoxy9- za9-a-methyl-9-ahomoerythromycin. The molecular weight is 749.0. Mode of action is inhibition of protein synthesis in bacteria by binding to 50 s ribosomal subunits and preventing translocation of peptides.

The advantages of Azithromycin for prophylaxis of RF/RHD are as follows:

> Its long half-life, requiring only once a week administration.

$>$ Safety profile.

$>$ Autoimmune suppressant property.

$>$ Suppressing inflammatory process in the heart.

$>$ It is effective orally, no degradation by the gastric acids.

$>$ It is well-absorbed, especially on an empty stomach.

> Peak concentration achieved in 2.1 to 3.2 hours when taken orally and in 1 hour with IV administration.

$>$ Due to its high concentration in phagocytes, the drug reaches the infection site. From the phagocytes, the drug is released in graded manner with large concentrations at the infected site.

> Due to ion trapping and high-lipid solubility in the plasma, the azithromycin concentration is 50 times higher than in tissues.

> The concentration of azithromycin in lungs and tonsils exceeds MIC 90 after a single dose of $500 \mathrm{mg}$, which is a significant advantage.

Due to its long half-life, it requires a single dose per day.

Able to maintain the bacteriostatic drug levels for several days in infected tissue.

After a single dose of $500 \mathrm{mg}$ of azithromycin, plasma concentration decreases in a polyphasic pattern with an elimination half-life of 68 hours. However, the prolonged half-life of the drug is due to the extensive uptake and graded release of drug from tissues.

If started with 500 mg daily for 5 consecutive days; in tissues, the drug persists for up to 6 days. For treatment prevention and cure of RF/RHD, our focus should be to eradicate the GABHS infection as early as possible.

For more than six decades, the various forms of penicillin and many other antibiotics have been tried. However, there is a massive disadvantage of antibiotics' daily administration for an indefinite period of several years, especially young patients.

The best drug for GABHS is azithromycin which, if started within 9 days of sore throat, can prevent almost all cases of ARF. ${ }^{2}$

Usually, the sore throat of viral or bacterial etiology resolves itself in 3 to 5 days. Even after symptomatic improvement, the full course of azithromycin $500 \mathrm{mg}(12 \mathrm{mg} / \mathrm{kg})$ once daily for 5 days should be given to prevent RF/RHD.

Secondary Prevention of RF: It is well-known that a patient who has had an attack of ARF is more prone to subsequent attacks, leading to progressive damage of the heart valves. An antibiotic effective against GABHS is essential to prevent organic heart valve disease by the culprit streptococci. For the past several decades, BPG 1.2 million units at intervals of 21 days has been the mainstay of treatment. Other antibiotics effective against group A streptococcus require daily dose.

Since 1991, after the discovery of azithromycin, it is the drug of choice for Group A Streptococcal (GAS) infections, and therefore it should be the drug of choice for RF/RHD because not to forget it is the causative organism, which should be the target of treatment.

Below is a list of reasons for replacing Penicillin with Azithromycin:

1. Today, healthcare workers avoid giving penicillin injections due to a fatal reaction in as much as 1 in 10000 among 20 lakh patients of RF/RHD in the world (AHA Scientific Statement Circulation March 24, 2009). ${ }^{3}$

2. BPG is unavailable and even sold at a very high cost in most developing countries.

3. The supply and demand ratio of benzathine penicillin is too low.

4. Penicillin injection can result in severe reaction even after several doses; therefore, sensitivity tests and consent are required each time.

5. When there is an option of a painless, risk-free, safe, readily available oral drug, it is unethical to use an older, toxic drug without offering the patient the choice. Today, it is not a "take or die" situation.

6. Penicillin injection is oily, painful, more than $2 \mathrm{~mL}$ in quantity, to be given deep intramuscular, and challenging to be administered to cachectic malnourished children.

7. Penicillin itself causes transient valvulitis of mitral and aortic valve after injection, so, ironically, this prompts the physician to continue the injections for another 5 years or even lifelong. ${ }^{4}$

8. Penicillin has interactions with drugs coprescribed in RF, that is, warfarin, aspirin, diuretics, etc.

9. Accidental IV injection can cause cardiorespiratory arrest and death.

10. Hypersensitivity and allergic reactions are common.

11. Pseudomembranous colitis occurs with repeated injections.

12. Clostridium difficile associated diarrhea (CDAD) occurs, which increases morbidity and mortality.

13. Penicillin causes pain, fever, swelling, and inflammation, which are itself the features of the disease RF, increasing the morbidity due to RF and even anaphylaxis, resulting in mortality. ${ }^{5}$

There is ample scientific supporting literature for all of the above. For the above reasons, the patient may be terrified of the treatment than the disease, which leads to a high percentage of noncompliance, resulting in relentless progress of the disease with irregular, painful prophylaxis. 


\section{The miracle solution is as follows:}

Tablet azithromycin $500 \mathrm{mg}$ once daily for 5 days consecutively as initial treatment followed by prophylaxis with tablet azithromycin $500 \mathrm{mg}$ once a week for 1 year only. In children, a single dose of $10 \mathrm{mg} / \mathrm{kg}$ or $20 \mathrm{mg} / \mathrm{kg}$ for 3 days only can be given with good efficacy. Above 5 years of age, a daily dose of 500 mg may be given.

Explanation: Given only once in a week, azithromycin is sufficient to eradicate GAS infections and suppress any autoimmune destructive process.

The inflammatory process may start in the heart valves within 9 days. If the drug is to be given weekly for prophylaxis, $500 \mathrm{mg}$ continued for 5days should be the starting dose to prolong the half-life of the drug. Up to 6 days, the drug persists in the tissues, if given according to this regime. Azithromycin may be continued for 1 year in case of any relapse recurrence, new infection, any symptoms or feature of $\mathrm{RF} / \mathrm{RHD}$, or even started again if any features of RF occur. However, each time, it should be started with $500 \mathrm{mg}$ for 5 consecutive days. Each time azithromycin is started or switched to it must be given in a dose of $500 \mathrm{mg}$ for 5 days. ${ }^{6}$

In our institution, this regime has been practiced for the past 9 years and found to be 100 percent effective in that no relapse recurrence or new cases of RF were found with $100 \%$ compliance. Azithromycin eradicates streptococci from the oropharynx and therefore results in cessation of the RHD process. It also arrests the valve destruction by streptococci by its immunosuppressant properties. It should be used with caution in renal failure but safe in moderate hepatic impairment. Azithromycin is safe in pregnancy.

In a study of azithromycin for ARF, 730 patients were given azithromycin $500 \mathrm{mg}$ daily for 5 days; thereafter, $500 \mathrm{mg}$ on 2 consecutive days in a week. With $100 \%$ compliance, there was no relapse, rebound rheumatic activity, reinfection, or worsening of valve disease in any patient. Patients who had no new problem suggestive of RF/RHD were given azithromycin prophylaxis for 1 year only. The study proved the effectiveness and compliance with azithromycin in RF/RHD. ${ }^{6}$

Dr Stephen Marko of World Heart Federation, University of Connecticut, expressed concern over the use of BPG in RF/RHD due to shortage and poor quality of drugs. He also agreed to tailor-made treatment guidelines to local requirements than stringent recommendations (personal communication to the author).?

In a retrospective review of 144 ARF patients, the recurrence rate was $38 \%$, mean compliance was $59 \%$ with BPG in patients without recurrence, and $57 \%$ in patients with recurrence, which proves that BPG is not an effective prophylaxis. ${ }^{8}$ The recurrence rate of RF with BPG is 3 to $8 \%$ over 5 to 6 years in various studies, but with azithromycin, it is less than $3 \%$ consistently.

To prevent a recurrence, a serum penicillin level of $>0.02$ $\mu \mathrm{gm} / \mathrm{mL}$ is required. However, with azithromycin, serum level is irrelevant, as it persists in tissues in high concentrations, and therefore it is more efficacious. Azithromycin, if started within 9 days of sore throat, can prevent all cases of RF/RHD. ${ }^{9}$
The general reluctance to switch from penicillin to a new drug is because of the presumption that penicillin is the only drug efficacious in RF/RHD and the gold standard. However, the question is, what is penicillin's role in RHD; after all, it is just used for its antistreptococcal efficacy, and that is all. Therefore, if a better drug with superior efficacy against GABHS is there without the disadvantages of penicillin and with which patient compliance be assured, should it not replace penicillin posthaste?

The rationale for giving azithromycin prophylaxis for only 1 year is that various studies have shown that it takes at least 2 weeks after a streptococcal infection for autoimmune destruction of heart valves, and it is doubtful that the bacteria or its products could cause RHD after more than 3 months of entry. Even in poststreptococcal reactive arthritis, valvular heart disease (VHD) is seen only for a few months after the streptococcal infection. The recommendation is for only 1 year of secondary prophylaxis in these patients (Class IIB, LOE C-AHA Scientific Statement) ${ }^{9}$

The present recommendation of the American Heart Association for the duration of prophylaxis with BPG is 5 years to lifelong after the attack with a footer that this can be modified according to circumstances. This footer makes it evident that the duration of treatment has been arbitrarily decided and time interval for causing autoimmune damage, time gap for causing heart valve destruction from carditis, persistence, disappearance, susceptibility of the bacteria, epidemiological factors, individual resistance, and comparison with other drugs have not been taken into consideration. ${ }^{10}$

A drug that has to be taken lifelong cannot be labeled prophylactic.

A drug that causes more risk to life than the disease cannot be used for prophylaxis.

There cannot be a better, more effective, safe long-acting oral drug for GAS than azithromycin (Class 1 LOE-C).

No doubt that for prophylaxis and treatment of RF/RHD, azithromycin wins hands down over BPG.

In a study at GSVM Medical College, Kanpur, azithromycin was used exclusively in RF/RHD in patients from in and around Kanpur belonging to the lower socioeconomic strata. The incidence of RF/RHD is approximately 4 per $1000 .{ }^{11}$

The use of BPG injection was considered unethical, dangerous, less efficacious, and even fatal in certain cases; therefore, studies with a direct comparison with azithromycin were not done, but the review of the literature showed azithromycin to be the best drug for GABHS. ${ }^{12}$

ARMOR: Azithromycin must be given in a dose of $500 \mathrm{mg}$ one tablet daily for 5 days, followed by one tablet once a week for 1 year for primary prevention, treatment and secondary prophylaxis of $\mathrm{RF} / \mathrm{RHD}$, respectively. ${ }^{13}$

\section{Conflicts of Interest}

None declared.

\section{References}

1 International rheumatic fever study group: allergic reactions to long-term benzathine penicillin prophylaxis for rheumatic fever, Lancet 1991;337:1308-1310 
2 Donde S, Mishra A, Kochhar P. Azithromycin in acute bacterial URTI: an Indian noninterventional study. Indian J Otolaryngol Head Neck Surg 2014;66(Suppl 1):225-230

3 Gerber MA, Baltimore RS, Eaton CB, et al. AHA Scientific Statement. Prevention of RF and diagnosis and treatment of acute streptococcal pharyngitis. Circulation 2009;119(11): 1541-1551

4 Thomson WO. Sudden death following injection penicillin. BMJ 1952;2(477):70-72

5 Altamimi S, Khalil A, Khalaiwi KA, Milner R, Pusic MV, Al Othman MA. Short versus standard duration antibiotic therapy for acute streptococcal pharyngitis in children. Cochrane Database Syst Rev 2009;(1):CD004872

6 Lalchandani A, Rana M, Mehrotra M, Prabhu K. Taruni Lalchandani: benzathine penicillin must be substituted with azithromycin for treatment and prophylaxis of RF. Circulation 2010;122e115 p712, https://doi.org/10.1161/ CIRCULATIONAHA.110.192774

7 Marko SB. Benzathine Penicillin G for the Prevention of Rheumatic Fever and Rheumatic Heart Disease in the
Deve-loping World: A Global Survey of the Quality and Quantity of Supply [master's theses]. CT, USA: UCONN Library, University of Connecticut Graduate School; 2014

8 Seckeler MD, Hoke TR, Gurka MJ, Barton LL. No demonstrable effect of benzathine penicillin on recurrence of rheumatic Fever in pacific island population. Pediatr Cardiol 2010;31(6):849-852

9 AHAscientificstatement.Circulation2009;119(11):1541-1551

10 Daniels ED, Mohanlal D, Pettifor JM. RF prophylaxis in SA. S Afr Med J 1994;84:477-481

11 Lalchandani A, Shameem M, Chandra S, et al. Use of alternative drugs for RF prophylaxis in place of injection benzathine penicillin. Indian Heart J 2005;57:462

12 Schaad UB. Acute streptococcal tonsillopharyngitis: a review of clinical efficacy and bacteriological eradication. J Int Med Res 2004;32(1):1-13

13 Azithromycin (ARMOR) must replace benzathine penicillin for treatment and prophylaxes of rheumatic fever. Available at: https://www.longdom.org/conference-abstracts-files/2155-9880.C1.036-019.pdf. Accessed January 1, 2021 